\title{
Stout Smearing for Twisted Mass Fermions
}

\author{
Karl Jansen* \\ NIC/DESY Zeuthen, Platanenallee 6, D-15738 Zeuthen, Germany \\ E-mail: karl.jansen@desy.de
}

\section{Craig McNeile}

Dept. of Physics and Astronomy, Kelvin Build., University of Glasgow, Glasgow G12 8QQ, UK

E-mail: C.mcneileephysics.gla.ac.uk

\section{István Montvay}

Deutsches Elektronen-Synchrotron DESY, Notkestr. 85, D-22607 Hamburg, Germany

E-mail: istvan.montvayedesy.de

\section{Chris Richards}

Theoretical Physics Div., Dept. of Mathematical Sciences, University of Liverpool,

Liverpool L69 7ZL, UK

E-mail: c.m.richardseliverpool.ac.uk

\section{Enno E. Scholz}

Physics Department, Brookhaven National Laboratory, Upton, NY, 11973, USA

E-mail: scholzee@quark.phy.bnl.gov

\section{Carsten Urbach ${ }^{\dagger}$}

Theoretical Physics Div., Dept. of Mathematical Sciences, University of Liverpool,

Liverpool L69 7ZL, UK

E-mail: Carsten.Urbach@liverpool.ac.uk

\section{Urs Wenger}

Institute for Theoretical Physics, ETH Zürich, CH-8093 Zürich, Switzerland

E-mail: wengerephys.ethz.ch

The effect of Stout smearing is investigated in numerical simulations with twisted mass Wilson quarks. The phase transition near zero quark mass is studied on $12^{3} \times 24,16^{3} \times 32$ and $24^{3} \times 48$ lattices at lattice spacings $a \simeq 0.1-0.125 \mathrm{fm}$.

The XXV International Symposium on Lattice Field Theory

July 30 - August 42007

Regensburg, Germany

\footnotetext{
* Speaker.

${ }^{\dagger}$ Current address: Humboldt-Universität zu Berlin, Institut für Physik, Newtonstr. 15, 12489 Berlin, Germany
} 
The phase structure of Wilson fermions with twisted mass $(\mu)$ has been investigated in [1, 2]. As it is explained there, the observed first order phase transition limits the minimal pion mass which can be reached in simulations at a given lattice spacing: $m_{\pi}^{\min } \simeq \mathscr{O}(a)$. The phase structure is schematically depicted in the left panel of Fig. 1. The phase transition can be observed in simulations with twisted mass fermions, for instance, as a "jump" or even metastabilities in the average plaquette value as a function of the hopping parameter $(\kappa)$. (The right panel of Fig. 1 shows thermal cycles to demonstrate this phenomenon.)

One possibility to weaken the phase transition and therefore allow for lighter pion masses at a given lattice spacing is to use an improved gauge action like the DBW2, Iwasaki, or tree-level Symanzik (tlSym) improved gauge action instead of the simple Wilson gauge action. This has been successfully demonstrated in [3, 4 , 5].

Here we report on our attempts to use a smeared gauge field in the fermion lattice Dirac operator to further reduce the strength of the phase transition. This is relevant in simulations with $N_{f}=2+1+1(u, d, s, c)$ quark flavours [6] where the first order phase transition becomes stronger compared to $N_{f}=2$ simulations. The main impact of the above mentioned improved gauge actions on the gauge fields occuring in simulations is to suppress short range fluctuations ("dislocations") and the associtated "exceptionally small" eigenvalues of the fermion matrix. The same effect is expected from smearing the gauge field links in the fermion action. The cumulated effect of the improved gauge action and smeared links should allow for a smaller pion mass at a given lattice spacing and volume. Our choice is the Stout smearing procedure as introduced in [7], since it can easily be implemented in the Hybrid Monte Carlo (HMC) based updating algorithms we are currently using.

One should keep in mind that a possible caveat of this procedure is "oversmearing", i.e., removing too many small eigenvalues by applying too many smearing steps and/or using a too high value for the smearing parameter-because not every small eigenvalue is "unphysical". In addition, after many smearing steps the fermion action can become too delocalised which can lead to an unwanted slowing down of the approach to the continuum limit. In order to avoid this caveat we choose to work with only one step of very mild Stout smearing. Moreover we keep these smearing parameters fixed as we change the lattice spacing.

In Section 11 we will shortly review the smearing procedure and the twisted mass formulation, as well as some details concerning the used updating algorithms. Section 2 is devoted to the presentation of the results of our numerical simulations using $N_{f}=2$ and $N_{f}=2+1+1$ flavours of twisted mass quarks.

\section{Stout smearing and twisted mass fermions}

\subsection{Analytic smearing for SU(3) link variables}

To have a smearing procedure which is analytic in the unsmeared link variables is an essential feature, if one is to use the smeared variables in an updating scheme like HMC based algorithms requiring the calculation of the derivative (or force) with respect to the unsmeared link variables. The Stout smearing procedure as introduced by Morningstar and Peardon in [7] was designed to meet this requirement. We will briefly describe it in the following but will limit ourselves to the case of SU(3). For more details we refer to the cited work. 

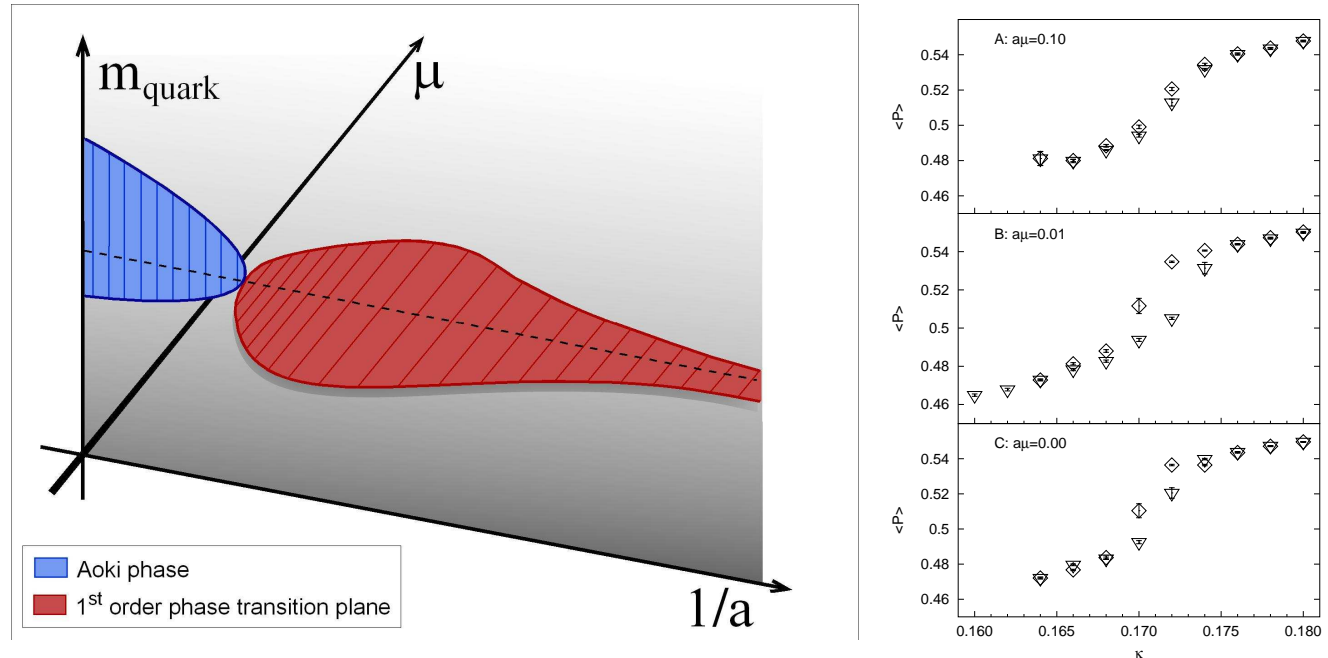

Figure 1: Left panel: The phase diagram with Wilson fermions. Right panel: Thermal cycles with $a \mu=0.1$, 0.01 , and without a twisted mass term on $8^{3} \times 16$ lattices at $\beta=5.2$ (Wilson gauge action), see [1].

The $(n+1)^{\text {th }}$ level of Stout smeared gauge links is obtained iteratively from the $n^{\text {th }}$ level by

$$
U_{\mu}^{(n+1)}(x)=e^{\mathrm{i} Q_{\mu}^{(n)}(x)} U_{\mu}^{(n)}(x) .
$$

In the following we will refer to the unsmeared ("thin") gauge field as $U_{\mu} \equiv U_{\mu}^{(0)}$, while the maximally smeared ("thick") gauge field for $N$-level Stout smearing will be denoted as $\widetilde{U}_{\mu} \equiv U_{\mu}^{(N)}$. The $\mathrm{SU}(3)$ matrices $Q_{\mu}$ are defined via the staples $C_{\mu}$ :

$$
\begin{aligned}
Q_{\mu}^{(n)}(x)= & \frac{\mathrm{i}}{2}\left[U_{\mu}^{(n)}(x) C_{\mu}^{(n)^{\dagger}}(x)-\text { h.c. }\right]-\frac{\mathrm{i}}{6} \operatorname{Tr}\left[U_{\mu}^{(n)}(x) C_{\mu}^{(n)^{\dagger}}(x)-\text { h.c. }\right], \\
C_{\mu}^{(n)}= & \sum_{v \neq \mu} \rho_{\mu v}\left(U_{v}^{(n)}(x) U_{\mu}^{(n)}(x+\hat{v}) U_{v}^{(n)^{\dagger}}(x+\hat{\mu})\right. \\
& \left.+U_{v}^{(n)^{\dagger}}(x-\hat{v}) U_{\mu}^{(n)}(x-\hat{v}) U_{v}^{(n)}(x-\hat{v}+\hat{\mu})\right),
\end{aligned}
$$

where in general $\rho_{\mu \nu}$ is the smearing matrix. In our numerical simulations we used exclusively isotropic 4-dimensional smearing, i.e., $\rho_{\mu v}=\rho$.

The thick gauge field will only be used in the fermion operator, cf. Eq. (1.7). Therefore the usage of smeared links is nothing else but a different discretization of the covariant derivative operator on the lattice. For the gauge part of the action the thin gauge field still is the relevant one.

\subsection{Twisted mass fermion action}

The notations in this subsection follow Ref. [6]. (For details we refer to this work.) We performed simulations with one light doublet $(u, d)$ of twisted mass Wilson fermions (only using the fermion matrix $\left.Q_{l}^{(\chi)}\right)$. Later on we shall add a second doublet for the heavier quarks $(c, s)$, where the masses are non-degenerate due to the addition of an extra mass term (cf. [8, 97). The 
fermion action then reads

$$
\begin{aligned}
S^{\text {fermion }} & =\sum_{x, y}\left(\bar{\chi}_{l, x} Q_{l, x y}^{(\chi)} \chi_{l, y}+\bar{\chi}_{h, x} Q_{h, x y}^{(\chi)} \chi_{h, y}\right), \\
Q_{l}^{(\chi)} & =\mu_{\kappa_{l}}+\mathrm{i} \gamma_{5} \tau_{3} a \mu_{l}+(N+R)_{x y}, \\
Q_{h}^{(\chi)} & =\mu_{\kappa_{h}}+\mathrm{i} \gamma_{5} \tau_{1} a \mu_{\sigma}+\tau_{3} a \mu_{\delta}+(N+R)_{x y}, \\
(N+R)_{x y} & =-\frac{1}{2} \sum_{\mu= \pm 1}^{ \pm 4} \delta_{x, y+\hat{\mu}} \widetilde{U}_{\mu}(y)\left(\gamma_{\mu}+r\right)
\end{aligned}
$$

where $\mu_{\kappa_{X}}=1 /\left(2 \kappa_{X}\right)$ is the untwisted mass and $a \mu_{l}, a \mu_{h}$, and $a \mu_{\delta}$ are the twisted mass terms in the light and heavy doublet and the split mass term, respectively.

\subsection{Algorithms}

We used two different algorithms with independent implementations of the Stout smearing routines to be able to cross-check our results. The first algorithm is the HMC algorithm with multiple time scale integration and mass preconditioning as described in [10]. In that case, the smearing routines were taken from the CHROMA-code package [11] and a chronological inverter was included, too.

Since this algorithm only allows to simulate an even number of fermion flavours (also excluding the case of a split doublet as described in Sec. 2.2), as a preparation for the $N_{f}=2+1+1$ simulations, we also added Stout smearing routines to our existing Polynomial HMC (PHMC) [12, 13] update code, where we perform one stochastic correction step at the end of a trajectory. For details on the implementation of the PHMC, cf. [14, 15]. We used trajectory lengths of $2 \times 0.35$ to $3 \times 0.35$ and determinant breakup of $n_{B}=2$.

\section{Numerical simulations}

\section{1 $N_{f}=2$}

In all of the simulations presented here, we used the tree-level Symanzik improved gauge action on either $12^{3} \times 24,16^{3} \times 32$, or $24^{3} \times 48$ lattices. We compare results obtained using one level of Stout smearing $(N=1)$ with $\rho=0.1$ or $\rho=0.125$ to simulations without smearing of the link variables in the Wilson twisted mass fermion action. Our choice of mild smearing (smearing only once with a small parameter) should guarantee that the fermion action remains well localized on physical scales even on relatively coarse lattice spacings.

Figures 2 and 3 show the average value of the (thin) plaquette without and with smearing, respectively. In the case without smearing a jump in the average plaquette value is clearly visible. Here we also observed metastabilities, which show up as differences between runs starting from a random (hot) or ordered (cold) configuration (red circles and blue triangles, respectively, in Fig. 2). In the case of Stout smearing it is unclear whether there is still a phase transition at all, since the left panel of Fig. 3 shows a rather smooth dependence of the average plaquette value on the inverse hopping parameter. To examine if metastabilities may still arise with Stout smearing, we started runs from either a random (hot) or ordered (cold) configuration at the same parameters where the 

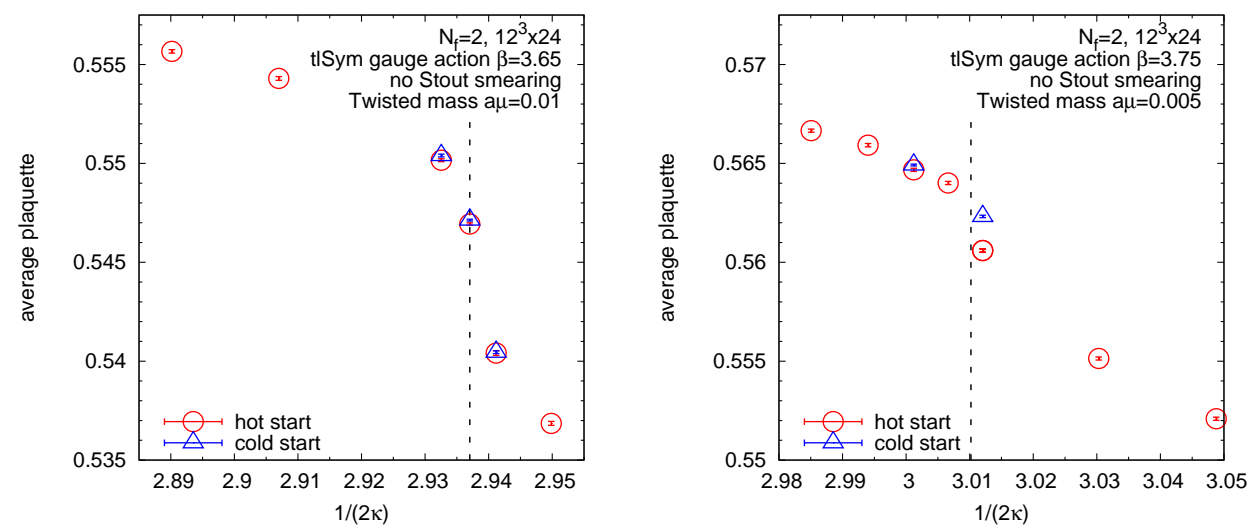

Figure 2: Average plaquette value without Stout smearing with hot (red circles) and cold starts (blue triangles) at two different values for the gauge coupling $\beta$ and twisted mass $a \mu$.
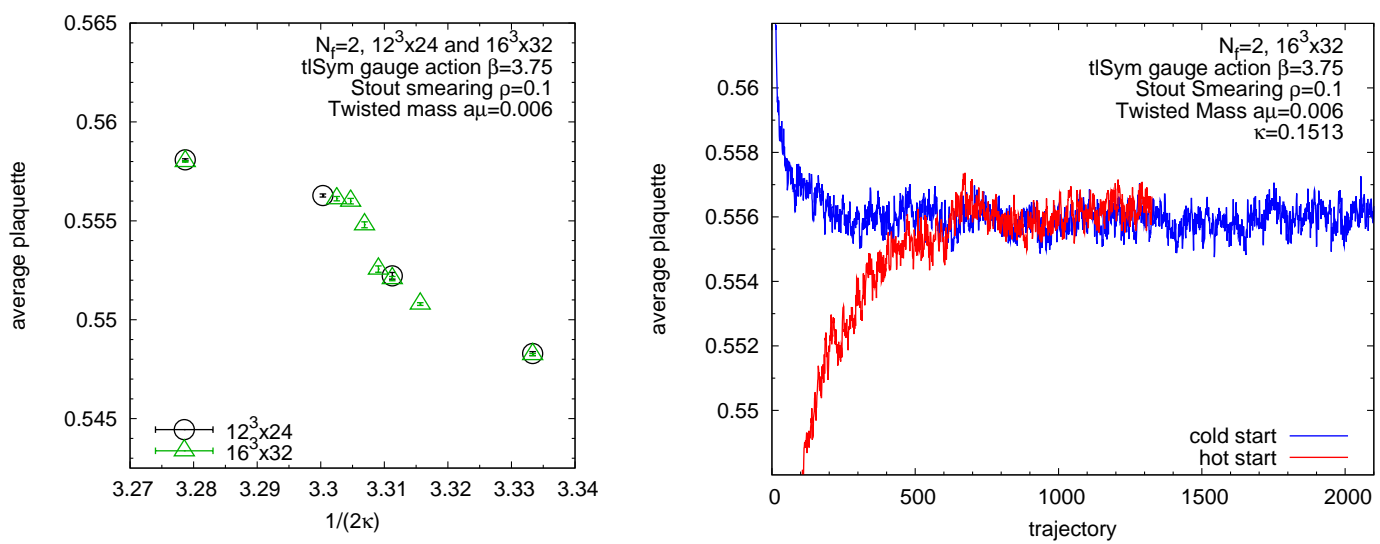

Figure 3: Simulations with Stout smearing. Left panel: Average plaquette value on two different lattice sizes. Right panel: Monte Carlo history of the plaquette value for hot (red) and cold (blue) starts.

hopping parameter was chosen to lie in the region of fastest increase of the average plaquette: $\kappa=0.1513$ or $1 /(2 \kappa) \approx 3.305$; the Monte Carlo histories of the two runs are displayed in the right panel of Fig. 3. One can see that after roughly 500 trajectories both runs thermalized at the same average value for the plaquette giving no evidence to the existence of metastabilities. Figure 4 shows the (untwisted) PCAC quark mass (left panel) and the squared pion mass (right panel) as a function of $\mu_{\kappa}$. The former also shows no clear evidence for the presence of a phase transition, since both branches (positive and negative PCAC quark mass) extrapolate to roughly the same critical value of $\mu_{\kappa}$. From the latter one can read off that on the volume $L / r_{0} \simeq 4$ a minimal pion mass of $m_{\pi} r_{0} \simeq 0.7$ is easily achieved for $a \approx r_{0} / 4$. (We use here for setting the scale the Sommer parameter $r_{0}$.)

On the $24^{3} \times 48$ lattice in a previous simulation at $\beta=3.8$ [16] without smearing we observed a problematic behaviour in time histories implying very long autocorrelations. We are presently repeating this run with one level of Stout smearing $(\rho=0.125)$ to see the effect of smearing for such a situation. Although our first results indicate that smearing helps, it is too early to give a definite conclusion at this point. It will also be interesting to compare physical observables, e.g., $f_{\mathrm{PS}}$ and $m_{\mathrm{PS}}$, between the Stout smeared and unsmeared simulations. 

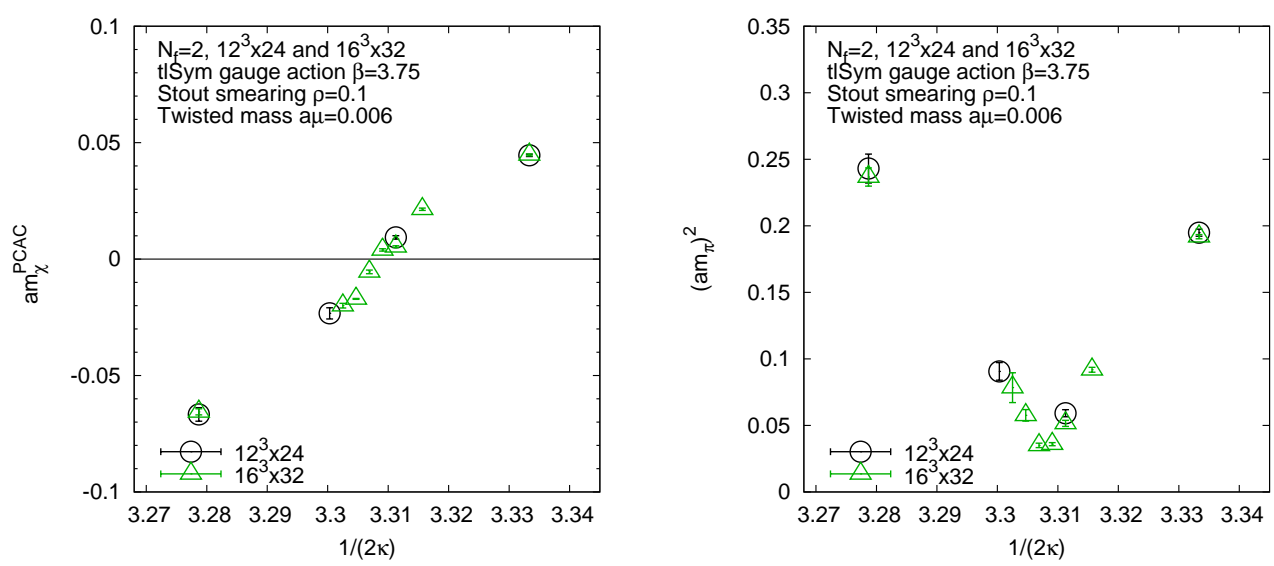

Figure 4: Left panel: PCAC quark mass, right panel: squared pion mass using Stout smearing.
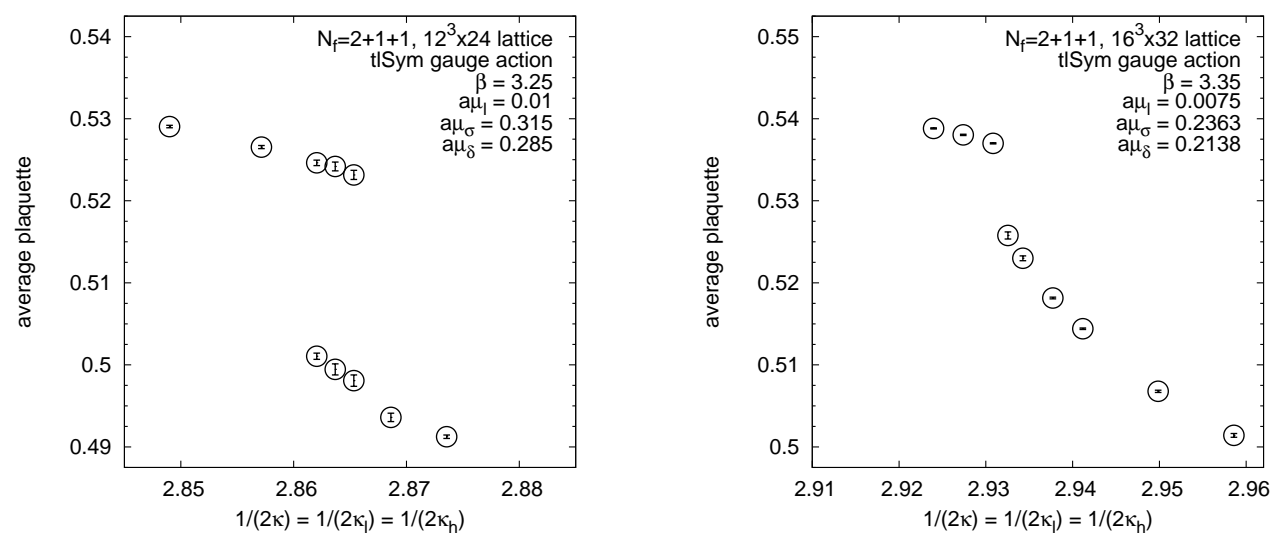

Figure 5: Average plaquette with $N_{f}=2+1+1$ flavours on $12^{3} \times 24, \beta=3.25$ (left panel) and $16^{3} \times 32$, $\beta=3.35$ (right panel) lattices, see [6].

2.2 $N_{f}=2+1+1$

Recently, the possibility of adding the strange quark in dynamical twisted mass simulations has been tried following the lines of [9] by introducing a mass splitting term in the heavier doublet, see Eq. (1.6). In that way not only a strange quark will be added but also the much heavier charm quark is taken into account. For first numerical results see [6], where an important conclusion is that the extra dynamical quarks strengthen the first order phase transition. As an example, in Fig. 5 we show the jump in the average plaquette for two different lattice spacings at a fixed physical volume. On the coarser lattice spacing (left panel) again metastabilities show up. At the finer lattice spacing (right panel) there are no more metastabilities but there is still a considerable "jump" in the average plaquette. The findings from our Stout smeared run for $N_{f}=2$ suggest that smearing could substantially help in the case of $N_{f}=2+1+1$.

\section{Conclusions \& Outlook}

The conclusion of testing Stout smearing with twisted mass Wilson quarks is that the first order phase transition at non-zero lattice spacing becomes weaker as a result of smearing. Therefore 
moderate Stout smearing can be an option-in particular for future numerical simulations in the twisted mass formalism with dynamical $u$-, $d$-, $s$ - and $c$-quarks.

Acknowledgements. We would like to thank S. Dürr for discussions. The numerical simulations were performed on the QCDOC at Edinburgh, the JUMP at Forschungszentrum Jülich, and the Scotgrid. This work has been supported in part by the EU Integrated Infrastructure Initiative Hadron Physics (I3HP) under contract RII3-CT-2004-506078. E.S. was supported by the U.S. Dept. of Energy under contract DE-AC02-98CH10886.

\section{References}

[1] F. Farchioni et al., Twisted mass quarks and the phase structure of lattice QCD, Eur. Phys. J. C39 (2005) 421-433 [hep-lat/0406039].

[2] F. Farchioni et al., Lattice spacing dependence of the first order phase transition for dynamical twisted mass fermions, Phys. Lett. B624 (2005) 324-333 [hep-lat/0506025].

[3] F. Farchioni et al., The phase structure of lattice QCD with Wilson quarks and renormalization group improved gluons, Eur. Phys. J. C42 (2005) 73-87 hep-lat/0410031.

[4] F. Farchioni et al., Numerical simulations with two flavours of twisted-mass Wilson quarks and DBW2 gauge action, Eur. Phys. J. C47 (2006) 453-472 [hep-lat/0512017].

[5] ETM Collaboration, P. Boucaud et al., Dynamical twisted mass fermions with light quarks, Phys. Lett. B650 (2007) 304-311 [hep-lat/0701012].

[6] T. Chiarappa et al., Numerical simulation of $Q C D$ with $u, d$, $s$ and $c$ quarks in the twisted-mass Wilson formulation, Eur. Phys. J. C50 (2007) 373-383 [hep-lat/0606011].

[7] C. Morningstar and M. J. Peardon, Analytic smearing of SU(3) link variables in lattice QCD, Phys. Rev. D69 (2004) 054501 [hep-lat/0311018].

[8] R. Frezzotti, P. A. Grassi, S. Sint and P. Weisz, A local formulation of lattice QCD without unphysical fermion zero modes, Nucl. Phys. Proc. Suppl. 83 (2000) 941-946 hep-lat/9909003.

[9] R. Frezzotti and G. C. Rossi, Twisted-mass lattice QCD with mass non-degenerate quarks, Nucl. Phys. Proc. Suppl. 128 (2004) 193-202 [hep-lat/0311008].

[10] C. Urbach, K. Jansen, A. Shindler and U. Wenger, HMC algorithm with multiple time scale integration and mass preconditioning, Comput. Phys. Commun. 174 (2006) 87-98 [hep-lat/0506011].

[11] SciDAC Collaboration, R. G. Edwards and B. Joó, The Chroma software system for lattice QCD, Nucl. Phys. Proc. Suppl. 140 (2005) 832 hep-lat/0409003,

[12] P. de Forcrand and T. Takaishi, Fast fermion Monte Carlo, Nucl. Phys. Proc. Suppl. 53 (1997) 968-970 [hep-lat/9608093].

[13] R. Frezzotti and K. Jansen, A polynomial hybrid Monte Carlo algorithm, Phys. Lett. $\mathbf{B 4 0 2}$ (1997) 328-334 hep-lat/9702016].

[14] I. Montvay and E. Scholz, Updating algorithms with multi-step stochastic correction, Phys. Lett. B623 (2005) 73-79 hep-lat/0506006.

[15] E. E. Scholz and I. Montvay, Multi-step stochastic correction in dynamical fermion updating algorithms, PoS LAT2006 (2006) 037 hep-lat/0609042.

[16] C. Urbach, Lattice QCD with two light Wilson quarks and maximally twisted mass, these proceedings. 